\title{
Renal Insufficiency and Early Bystander CPR Predict In-Hospital Outcomes in Cardiac Arrest Patients Undergoing Mild Therapeutic Hypothermia and Cardiac Catheterization: Return of Spontaneous Circulation, Cooling, and Catheterization Registry (ROSCCC Registry)
}

\author{
Anjala Chelvanathan, ${ }^{1}$ David Allen, ${ }^{1}$ Hilary Bews, ${ }^{2}$ John Ducas, ${ }^{1}$ \\ Kunal Minhas, ${ }^{1}$ Minh Vo, ${ }^{1}$ Malek Kass, ${ }^{1}$ Amir Ravandi, ${ }^{1,2}$ James W. Tam, ${ }^{1}$ \\ Davinder S. Jassal, ${ }^{1,2,3,4}$ and Farrukh Hussain ${ }^{1}$ \\ ${ }^{1}$ Section of Cardiology, Department of Internal Medicine, University of Manitoba, Winnipeg, MB, Canada R2H $2 A 6$ \\ ${ }^{2}$ Institute of Cardiovascular Sciences, St. Boniface Research Centre, University of Manitoba, Winnipeg, MB, Canada R2H $2 A 6$ \\ ${ }^{3}$ Section of Oncology, Department of Internal Medicine, University of Manitoba, Winnipeg, MB, Canada R2H $2 A 6$ \\ ${ }^{4}$ Department of Radiology, University of Manitoba, Winnipeg, MB, Canada R2H $2 A 6$
}

Correspondence should be addressed to Davinder S. Jassal; djassal@sbgh.mb.ca

Received 26 September 2015; Revised 22 November 2015; Accepted 16 December 2015

Academic Editor: Firat Duru

Copyright (C) 2016 Anjala Chelvanathan et al. This is an open access article distributed under the Creative Commons Attribution License, which permits unrestricted use, distribution, and reproduction in any medium, provided the original work is properly cited.

\begin{abstract}
Objective. Out of hospital cardiac arrest (OHCA) patients are a critically ill patient population with high mortality. Combining mild therapeutic hypothermia (MTH) with early coronary intervention may improve outcomes in this population. The aim of this study was to evaluate predictors of mortality in OHCA patients undergoing MTH with and without cardiac catheterization. Design. A retrospective cohort of OHCA patients who underwent $\mathrm{MTH}$ with catheterization $(\mathrm{MTH}+\mathrm{C})$ and without catheterization (MTH + NC) between 2006 and 2011 was analyzed at a single tertiary care centre. Predictors of in-hospital mortality and neurologic outcome were determined. Results. The study population included 176 patients who underwent MTH for OHCA. A total of 66 patients underwent cardiac catheterization $(\mathrm{MTH}+\mathrm{C})$ and 110 patients did not undergo cardiac catheterization $(\mathrm{MTH}+\mathrm{NC})$. Immediate bystander CPR occurred in approximately half of the total population. In the MTH $+\mathrm{C}$ and $\mathrm{MTH}+\mathrm{NC}$ groups, the inhospital mortality was $48 \%$ and $78 \%$, respectively. The only independent predictor of in-hospital mortality for patients with MTH + $\mathrm{C}$, after multivariate analysis, was baseline renal insufficiency $(\mathrm{OR}=8.2,95 \% \mathrm{CI} 1.8-47.1$, and $p=0.009)$. Conclusion. Despite early cardiac catheterization, renal insufficiency and the absence of immediate CPR are potent predictors of death and poor neurologic outcome in patients with OHCA.
\end{abstract}

\section{Introduction}

Over 350,000 out of hospital cardiac arrests (OHCA) occur each year in the United States, with around 45,000 cardiac arrests occurring each year in Canada [1]. Despite nearly 40 years of promotion of prehospital Advanced Cardiac Life Support (ACLS), long-term survival rates following OHCA remain dismal $[2,3]$. In a recent meta-analysis, the aggregate survival rate for OHCA across various populations was reported at less than 8\% [3]. In addition, postcardiac arrest complications, including severe anoxic brain injury (ABI), contributes to high morbidity and mortality rates for patients initially undergoing successful resuscitation [1]. As such, it remains an important goal to develop therapeutic strategies to improve survival in this patient population.

Mild therapeutic hypothermia (MTH) and targeted temperature management (TTM) afford long-term survival and 
neurologic benefits to comatose survivors of arrhythmiainduced cardiac arrest $[1,3-6]$. More recently, studies have investigated the combination of early interventional strategies with MTH as a means of further improving long-term survival in resuscitated cardiac arrest patients with evolving evidence of ST elevation myocardial infarction (STEMI). A review of four nonrandomized case series involving STEMI patients who were successfully resuscitated from cardiac arrest and treated with both MTH and early coronary intervention demonstrated overall favorable outcomes [710]. Numerous other case series have illustrated that early coronary angiography and percutaneous coronary intervention (PCI), combined with MTH, produce the highest longterm survival rates among patients who remain comatose after resuscitated cardiac arrest [11-14]. As a result, in the ACCF/AHA 2013 STEMI Guidelines, MTH was endorsed as a class 1 , level of evidence $B$ recommendation [15]. Furthermore, immediate angiography and PCI as indicated were recommended in resuscitated OHCA patients whose initial ECG shows ST-segment elevation [15].

The objective of this retrospective study was to investigate the outcomes and predictors of mortality in individuals resuscitated from cardiac arrest who underwent MTH both with and without cardiac catheterization.

\section{Methods}

2.1. Study Design. A single-centre retrospective cohort study examining all consecutive adult patients who were successfully resuscitated following cardiac arrest who underwent either MTH with catheterization $(\mathrm{MTH}+\mathrm{C})$ or MTH without cardiac catheterization ( $\mathrm{MTH}+\mathrm{NC}$ ) between January 1, 2006, and September 30, 2011, was performed. MTH was used at the discretion of the attending physician and the decision regarding need for cardiac catheterization was made by the treating cardiologist or intensivist. MTH was induced and maintained through a combination of surface cooling blankets and ice packs. Local institutional protocol stipulates that MTH should be initiated as soon as possible after return of spontaneous circulation (ROSC) to achieve a core temperature of $32-34^{\circ} \mathrm{C}$ within $6-8 \mathrm{hrs}$. This core temperature should be maintained for $24 \mathrm{hrs}$ and followed by subsequent passive rewarming. The study was approved by the University of Manitoba and St. Boniface General Hospital research ethics boards.

2.2. Data Collection. Subjects were identified by searching the ICU quality-assurance database for an International Classification of Diseases-9 discharge diagnosis of cardiac arrest in addition to cointerrogation of the MACLAB cardiac catheterization database. Detailed demographics and prehospital data pertaining to the cardiac arrest, including location of cardiac arrest, duration of down time, bystander CPR, time to ROSC, and initial arrest rhythm, were collected. As cardiac rhythm monitoring was not always available during the pulseless period, the initial arrest rhythm was the first rhythm recorded either during or after the cardiac arrest. Cooling protocol and time data were noted. Detailed data on cardiac interventions were recorded, including coronary anatomy, stent placement, coronary artery bypass grafting (CABG), mechanical circulatory support (MCS), and intraaortic balloon pump (IABP) placement. Detailed data on complications within 1 week of hospitalization, in addition to patient outcomes, including in-hospital mortality, were abstracted.

2.3. Definitions. Cardiac arrest was defined as the absence of signs of circulation with the concomitant appearance of unconsciousness, apnea, or gasping and receipt of chest compressions or defibrillation for a pulseless arrhythmia as determined by a health care worker. Multivessel coronary disease was defined as $\geq 2$ main (left anterior descending, right coronary artery, or circumflex) vessels with $\geq 70 \%$ stenosis. Early coronary angiogram was defined as receiving a coronary angiogram within 12 hours of arrival. A successful PCI was defined as residual stenosis $\leq 20 \%$ with TIMI 3 flow. Slow flow was defined as TIMI $\leq 2$ flow in the intervened vessel. Angiographic, TIMI flow, and procedural success analysis was performed by local angiographic operators according to the aforementioned set criteria; there was no core lab available for analysis. The decision for PCI or CABG was at the operator's discretion, given the nature of a retrospective analysis.

Left ventricular ejection fraction (LVEF) was quantified using standard two-dimensional transthoracic echocardiography (TTE) or left ventriculography, whichever was available closer to index catheterization. The timing for TTE was variable, as this was a retrospective analysis and could include pre- or postrevascularization studies.

Cardiogenic shock was defined as systolic blood pressure $(\mathrm{SBP}) \leq 90 \mathrm{mmHg}$ for $>30$ minutes or the requirement of vasopressor/inotropic support to maintain SBP > $90 \mathrm{mmHg}$ in addition to evidence of end organ hypoperfusion. Baseline renal insufficiency was defined as creatinine clearance $(\mathrm{CrCl})<60 \mathrm{~mL} / \mathrm{min}$. $\mathrm{CrCl}$ was calculated using the standard Cockgroft-Gault equation $(\mathrm{CrCl}=(140-$ age $) \times$ wt $(\mathrm{kg})$ $\times F /($ plasma creatinine $\times 0.8136)$, where $F=1$ if male and 0.85 if female). Report of anoxic brain injury (ABI) required chart documentation by the intensivist or neurologist in the ICU and/or CT brain evidence of ABI with a clinical agreement note.

The Glasgow-Pittsburgh Cerebral Performance Category (CPC) score was utilized to assess neurologic recovery [16]. The best CPC score achieved at hospital discharge was recorded by the treating neurologist or allied health personnel. A CPC score of 1 or 2 represented favorable functional neurologic recovery and was therefore defined as good neurologic outcome. A CPC score of 3, 4, or 5 reflected poor neurologic recovery.

2.4. Statistics. Descriptive statistical methods were used to summarize data. A negative binomial logistic regression model was utilized to identify univariate and multivariate predictors of in-hospital mortality. All univariate predictors with a $p$ value $<0.05$ were considered significant and were included into a stringent multivariable model to prevent 
TABLE 1: Baseline clinical characteristics, prehospital arrest data, and cooling protocol of total population $(n=176)$.

\begin{tabular}{|c|c|c|c|}
\hline Clinical characteristics & $\mathrm{MTH}+\mathrm{C}(n=66)$ & $\mathrm{MTH}+\mathrm{NC}(n=110)$ & $p$ value \\
\hline Age (yrs) & $61 \pm 12$ & $61 \pm 16$ & 1.00 \\
\hline $\operatorname{Sex}(M)$ & $52(79)$ & $77(70)$ & 0.22 \\
\hline \multicolumn{4}{|l|}{ Medical history } \\
\hline Diabetes (\%) & $12(18)$ & $34(31)$ & 0.06 \\
\hline Smoking (\%) & $36(55)$ & $49(45)$ & 0.22 \\
\hline Hypertension (\%) & $36(55)$ & $65(59)$ & 0.63 \\
\hline Dyslipidemia (\%) & $27(41)$ & $39(35)$ & 0.42 \\
\hline Prior MI (\%) & $22(33)$ & $32(29)$ & 0.32 \\
\hline Prior PCI (\%) & $5(8)$ & $7(6)$ & 0.63 \\
\hline Baseline CRI (\%) & $17(26)$ & $12(11)$ & 0.24 \\
\hline \multicolumn{4}{|l|}{ Cardiac arrest } \\
\hline OHCA (\%) & $50(76)$ & $89(81)$ & 0.44 \\
\hline Witnessed OHCA (\%) & $61(92)$ & $83(75)$ & 0.07 \\
\hline Immediate bystander CPR (\%) & $36(55)$ & $40(45)$ & 0.20 \\
\hline Duration of bystander CPR (min) & $6.4 \pm 4.1$ & $7.5 \pm 4.0$ & 0.08 \\
\hline Time to ROSC from collapse (min) & $28.0 \pm 14.6$ & $24.0 \pm 14.8$ & 0.10 \\
\hline Time from collapse to EMS (min) & $8.0 \pm 5.0$ & $12.0 \pm 12.0$ & $<0.05$ \\
\hline Total cooled time (min) & $1671 \pm 410$ & $1625 \pm 551$ & 0.62 \\
\hline \multicolumn{4}{|l|}{ Initial arrest rhythm } \\
\hline $\mathrm{VF} /$ pulseless VT & $61(92)$ & $39(35)$ & $<0.05$ \\
\hline PEA & $5(8)$ & $61(65)$ & $<0.05$ \\
\hline STEMI & $45(68)$ & $0(0)$ & $<0.05$ \\
\hline \multicolumn{4}{|l|}{ Mild therapeutic hypothermia } \\
\hline Time from ROSC to cooling (min) & $277 \pm 110$ & $211 \pm 146$ & $<0.05$ \\
\hline Time to achieve $32-34^{\circ} \mathrm{C}$ from cooling (min) & $252 \pm 174$ & $312 \pm 466$ & 0.30 \\
\hline Total cooled time ( $\mathrm{min})$ & $1671 \pm 410$ & $1625 \pm 551$ & 0.56 \\
\hline
\end{tabular}

Values are mean \pm SD or $n(\%)$. MTH + C, mild therapeutic hypothermia with cardiac catheterization; OHCA, out of hospital cardiac arrest; MTH + NC, mild therapeutic hypothermia with no cardiac catheterization; yrs, years; m, males; MI, myocardial infarction; PCI, percutaneous coronary intervention; CRI, chronic renal insufficiency; CPR, cardiopulmonary resuscitation; min, minutes; ROSC, return of spontaneous circulation; EMS, emergency medical services; VF, ventricular fibrillation; VT, ventricular tachycardia; PEA, pulseless electrical activity; STEMI, ST elevation myocardial infarction.

model instability given our small sample size. SAS version 9.1.2 software was utilized to perform all analyses. Prespecified subgroup analyses were performed to identify univariate and multivariate predictors of good neurologic outcome.

\section{Results}

3.1. Study Population. A total of 176 consecutive patients (mean age $61 \pm 13$ years, 129 males) with a documented cardiac arrest who were admitted to a single tertiary care ICU and underwent MTH were the initial study population. A total of 66 patients underwent $\mathrm{MTH}+\mathrm{C}$ at the discretion of the treating cardiologist or intensivist; the remaining 110 patients did not undergo cardiac catheterization (MTH + NC). Baseline demographics of the two study groups are outlined in Table 1. Despite the majority of patients in the $\mathrm{MTH}+\mathrm{C}$ group having experienced a witnessed cardiac arrest (92\%), only half of patients (55\%) received immediate bystander CPR. Similarly, in the MTH + NC group, approximately $75 \%$ experienced a cardiac arrest, of which $45 \%$ received immediate bystander CPR. The mean duration of bystander CPR was similar at $6.4 \pm 4.1$ minutes and $7.5 \pm$
4.0 minutes for the MTH + C and MTH + NC groups, respectively. The median interval from the occurrence of cardiac arrest to ROSC was also similar at $28 \pm 15$ minutes and $24 \pm 15$ minutes for the MTH $+\mathrm{C}$ and $\mathrm{MTH}+\mathrm{NC}$ groups, respectively. In the majority of patients who underwent $\mathrm{MTH}$ $+\mathrm{C}$, ventricular fibrillation (VF)/pulseless ventricular tachycardia (VT) (92\%) was the initial resuscitated cardiac rhythm, with $68 \%$ demonstrating evidence of STEMI. In patients who underwent $\mathrm{MTH}+\mathrm{NC}$, the initial cardiac rhythm was PEA or asystole in $65 \%$ of cases. The majority of patients in the entire population $(>80 \%)$ were in cardiogenic shock requiring vasopressor or inotropic support within the first week of resuscitation following cardiac arrest. Baseline renal insufficiency was present in $26 \%$ of patients in the $\mathrm{MTH}+$ $\mathrm{C}$ group as compared to $11 \%$ in patients in the $\mathrm{MTH}+\mathrm{NC}$ group $(p=0.8)$.

3.2. Mild Therapeutic Hypothermia (MTH). The parameters for MTH are listed in Table 1. The mean time from ROSC to initiation of MTH was $277 \pm 110$ minutes $(4.6 \pm 1.8 \mathrm{hrs})$ for the catheterization group and $211 \pm 146$ minutes $(3.5 \pm 2.4 \mathrm{hrs})$ for the no catheterization group. An average time of $252 \pm 174$ 
TABLE 2: Cardiac catheterization findings in study population who underwent $\mathrm{MTH}+\mathrm{C}(n=66)$.

\begin{tabular}{lc}
\hline Cardiogenic shock & $53(80)$ \\
$\quad$ Vasopressors & $53(80)$ \\
Inotropes & $26(40)$ \\
$\quad$ Duration of support (min) & $52 \pm 73$ \\
\hline IABP use & $24(36)$ \\
\hline ECMO use & $3(5)$ \\
\hline Early catheterization (<12 hrs) & $56(86)$ \\
\hline Time to catheterization from ROSC (min) & $290 \pm 333$ \\
$\quad$ STEMI (min) & $212 \pm 94$ \\
$\quad$ No STEMI (min) & $465 \pm 140$ \\
\hline (1) Vessel CAD & $24(36)$ \\
(2) Vessel CAD & $16(24)$ \\
(3) Vessel CAD & $14(21)$ \\
Branch vessel disease or no culprit & $12(18)$ \\
\hline PCI & $45(68)$ \\
\hline Multivessel PCI & $10(22)$ \\
\hline Stent deployment & $43(96)$ \\
\hline Number of stents utilized & $1.7 \pm 1.1$ \\
\hline Stent thrombosis & $2(4)$ \\
\hline Successful PCI & $40(89)$ \\
\hline Mean TIMI flow pre (min) & $1.4 \pm 1.4$ \\
Mean TIMI flow post (min) & $23(51)$ \\
\hline CABG & $2.9 \pm 0.6$ \\
\hline GPIIbIIIa inhibition & \\
\hline Values are mean \pm SD or $n$ (\%). IABP, intra-aortic balloon pump; ECMO, \\
extracorporeal membrane oxygenation; ROSC, return of spontaneous cir- \\
culation; STEMI, ST elevation myocardial infarction; CAD, coronary artery \\
disease; PCI, percutaneous coronary intervention; TIMI, thrombolysis in \\
myocardial infarction; CABG, coronary artery bypass grafting. & \\
\hline &
\end{tabular}

minutes $(4.2 \pm 2.9 \mathrm{hrs})$ and $312 \pm 465$ minutes $(5.2 \pm 7.8 \mathrm{hrs})$ for the $\mathrm{MTH}+\mathrm{C}$ and $\mathrm{MTH}+\mathrm{NC}$ groups, respectively, from cooling initiation was required to achieve the target cooling temperature between 32 and $34^{\circ} \mathrm{C}$. Total time at target cooling temperature was $1671 \pm 410$ minutes $(27.9 \pm 6.8 \mathrm{hrs})$ for the $\mathrm{MTH}+\mathrm{C}$ group and $1625 \pm 551$ minutes $(27.1 \pm 9.2 \mathrm{hrs})$ for the MTH + NC group. MTH was initiated prior to cardiac catheterization in only $27 \%$ of patients in the $\mathrm{MTH}+\mathrm{C}$ group.

3.3. Cardiac Interventions. Table 2 lists the cardiac interventions for the $\mathrm{MTH}+\mathrm{C}$ patient population. Early cardiac catheterization $(<12 \mathrm{hrs})$ was performed in the majority $(86 \%)$ of patients. Although the overall mean time to catheterization from ROSC was $290 \pm 333$ minutes, STEMI patients received emergency coronary angiography sooner than patients without ECG changes (212 \pm 94 minutes versus $465 \pm 140$ minutes). PCI was performed in two-thirds of patients and successful PCI was achieved in the majority of these cases $(89 \%)$.

3.4. Outcomes. In patients who underwent MTH with and without cardiac catheterization, the in-hospital mortality was $48 \%$ and $78 \%$, respectively. Among survivors in the $\mathrm{MTH}+\mathrm{C}$
TABLE 3: In-hospital outcomes for total population $(n=176)$.

\begin{tabular}{lccc}
\hline Clinical characteristics & $\begin{array}{c}\text { MTH + C } \\
(n=66)\end{array}$ & $\begin{array}{c}\text { MTH + NC } \\
(n=110)\end{array}$ & $p$ value \\
\hline $\begin{array}{l}\text { In-hospital mortality (\%) } \\
\text { Discharged home (\%) }\end{array}$ & $32(48)$ & $86(78)$ & $<0.05$ \\
$\begin{array}{l}\text { Discharged to long term } \\
\text { facility (\%) }\end{array}$ & $12(20)$ & $5(3)$ & $<0.05$ \\
$\begin{array}{l}\text { CPC 1-2 neurological } \\
\text { recovery (\%) }\end{array}$ & $32(48)$ & $10(9)$ & $<0.05$ \\
$\begin{array}{l}\text { CPC 3-5 neurological } \\
\text { recovery (\%) }\end{array}$ & $34(52)$ & $100(91)$ & $<0.05$ \\
$\begin{array}{l}\text { Length of hospital stay } \\
\text { (days) }\end{array}$ & $12 \pm 14$ & $8 \pm 8$ & $<0.05$ \\
$\begin{array}{l}\text { Length of ICU stay } \\
\text { (days) }\end{array}$ & $7 \pm 6$ & $5 \pm 5$ & 0.35 \\
\hline
\end{tabular}

Values are mean $\pm \mathrm{SD}$ or $n(\%)$. MTH $+\mathrm{C}$, mild therapeutic hypothermia with cardiac catheterization; MTH $+\mathrm{NC}$, mild therapeutic hypothermia with no cardiac catheterization; CPC, cerebral performance category; ICU, intensive care unit.

TABLE 4: Univariate predictors of in-hospital mortality for study population $(n=176)$.

\begin{tabular}{lcc}
\hline Clinical characteristics & $\begin{array}{c}\text { MTH }+ \text { C } \\
(n=66)\end{array}$ & $\begin{array}{c}\text { MTH + NC } \\
(n=110)\end{array}$ \\
\hline Age & 0.04 & 0.4 \\
Diabetes & 0.02 & 0.4 \\
Dyslipidemia & 0.02 & 0.6 \\
Baseline CRI & 0.003 & 0.6 \\
Cardiogenic shock & 0.05 & 0.04 \\
$\begin{array}{l}\text { Absence of cooling prior to } \\
\text { cardiac catheterization }\end{array}$ & 0.04 & N/A \\
Use of dobutamine & 0.04 & 0.06 \\
\hline
\end{tabular}

$\mathrm{MTH}+\mathrm{C}$, mild therapeutic hypothermia with cardiac catheterization; MTH $+\mathrm{NC}$, mild therapeutic hypothermia with no cardiac catheterization; CRI, chronic renal insufficiency.

group, only $48 \%$ of patients (32/66 patients) had good (CPC $1-2)$ neurologic recovery. On the contrary, among survivors in the MTH + NC group, only $13 \%$ of patients (14/110 patients) had good (CPC 1-2) neurologic recovery. Of those who survived in the MTH + C group, $20 \%$ patients were transferred to rehabilitation or referring hospital and one-third of the patients were successfully discharged home (Table 3). In the $\mathrm{MTH}+\mathrm{NC}$ group, only $5 \%$ were transferred to a long-term facility, and only $3 \%$ were successfully discharged home.

3.5. Predictors of In-Hospital Mortality. Univariate predictors of in-hospital mortality for patients with MTH + C included increasing age, diabetes, dyslipidemia, baseline renal insufficiency postcardiac arrest, cardiogenic shock, use of dobutamine, and failure to initiate MTH prior to catheterization (Table 4). Multivariate analysis of these predictors identified baseline renal insufficiency as the only independent predictor of in-hospital mortality in patients with $\mathrm{MTH}+\mathrm{C}(\mathrm{OR}=$ 8.2, 95\% CI 1.8-47.1, $p=0.009)$. In patients with $\mathrm{MTH}+$ 
TABLE 5: Univariate negative predictors of good neurologic outcome in study population who underwent $\mathrm{MTH}+\mathrm{C}(n=66)$.

\begin{tabular}{lc}
\hline Variables & $p$ value \\
\hline Diabetes & 0.03 \\
Absence of immediate CPR & 0.03 \\
Collapse to ROSC time & 0.02 \\
Baseline renal insufficiency & 0.006 \\
Baseline pH & 0.03 \\
No cooling implemented before catheterization & 0.02 \\
Seizure & 0.007 \\
\hline
\end{tabular}

$\mathrm{MTH}+\mathrm{C}$, mild therapeutic hypothermia with cardiac catheterization; CPR, cardiac pulmonary resuscitation; ROSC, return of spontaneous circulation.

NC, shock was the only independent predictor of in-hospital mortality $(\mathrm{OR}=3.5,95 \%$ CI 1.0-11.3, $p=0.04)$.

3.6. Predictors of Neurologic Outcome. Diabetes, absence of immediate bystander CPR, increased collapse to ROSC time, baseline renal insufficiency, lower baseline $\mathrm{pH}$, postcardiac arrest seizure, and failure to initiate MTH prior to catheterization were univariate negative predictors of good (CPC 12) neurologic outcome in patients with $\mathrm{MTH}+\mathrm{C}$ (Table 5). Multivariate analysis identified baseline renal insufficiency $(\mathrm{OR}=0.15,95 \% \mathrm{CI} 0.02-0.71, p=0.03)$ and the absence of immediate bystander CPR (OR $=0.22,95 \%$ CI $0.05-0.9, p=$ 0.04 ) as independent negative predictors of good neurologic outcome in patients with $\mathrm{MTH}+\mathrm{C}$. Univariate analysis for predictors of neurological outcome in patients with MTH + NC was not performed due to the small sample size of $n=10$ who had good neurological outcome (CPC 1-2) in this study group.

\section{Discussion}

The current study describes the outcomes and predictors of increased mortality for patients resuscitated from cardiac arrest and treated with the combination of MTH and catheterization versus MTH with no cardiac catheterization. In-hospital mortality for the study population of MTH $+\mathrm{C}$ was high at $48 \%$, with $97 \%$ of patient mortality attributed to brain death, as is typical for patients with $\operatorname{ROSC}[1,3$, 4]. Baseline renal insufficiency following resuscitation from cardiac arrest was determined to be the only independent predictor of in-hospital mortality in patients with MTH + C. Furthermore, independent negative predictors of neurologic outcome were determined to be baseline renal insufficiency and absence of immediate bystander CPR for patients with $\mathrm{MTH}+\mathrm{C}$.

Neurological outcomes after cardiac arrest are traditionally dismal. The poor prognosis is attributed to postcardiac arrest syndrome, which encompasses systemic ischemicreperfusion injury with subsequent biochemical, structural, and functional insult $[17,18]$. Ultimately, this leads to progressive cell destruction, postcardiac arrest brain injury, circulatory dysfunction, multiorgan failure, and death $[17,18]$. The beneficial effects of MTH are based on the prevention of this cascade, specifically, by reducing cellular metabolic needs and inhibiting temperature-sensitive pathways of the ischemiareperfusion cascade to slow ongoing hypoxic neurological insult $[13,17,18]$. As such, a number of studies have demonstrated improved neurological outcomes with the use of MTH in the postcardiac arrest period. In a multicentre blinded randomized control trial involving patients resuscitated after cardiac arrest due to VT, 55\% patients randomized to receive MTH had a favorable neurologic outcome, compared to 39\% in the control group [5]. A recent study by Neilsen et al. also demonstrated that targeted temperature management at $36^{\circ} \mathrm{C}$ after OHCA conferred a similar benefit to MTH from a neurological function standpoint [6]. Our study corroborates that individuals who survive aggressive postresuscitative care are neurologically intact, with $48 \%$ of survivors having favorable neurological function at hospital discharge. The preservation of neurological function is an important treatment goal, as survival is correlated with neurologic status [13].

In addition to $\mathrm{MTH}$, the role of early invasive strategies for postcardiac arrest patients has gained recent attention. In previous studies, $40-57 \%$ of OHCA patients without STsegment elevation had pathological findings with therapeutic options on coronary angiography $[11,19]$. A recent retrospective study involving a cohort of 435 patients with OHCA of presumed cardiac origin reported the poor predictive value of ST-segment elevation for coronary occlusion in the setting of cardiac arrest [11]. Furthermore, successful immediate coronary angioplasty was associated with a survival benefit regardless of ECG findings, suggesting that immediate catheterization may be warranted in the setting of resuscitated cardiac arrests even in the absence of STsegment elevation [11]. This has led to an increased adoption of emergency coronary angiography for all patients with OHCA of suspected cardiac origin [20,21]. In the current study of patients who underwent MTH $+\mathrm{C}, 32 \%$ did not have ST-segment elevation, $32 \%$ did not undergo PCI, and $18 \%$ had angiographically normal coronary arteries or nonocclusive coronary artery disease. Only patients with ST-segment elevation underwent emergent PCI after invasive angiography, raising question to the use of universal emergent angiography postcardiac arrest. This is an important consideration as there are inherent risks associated with coronary angiography and the ultimate goal is to reduce the number of patients undergoing an unnecessary procedure.

A contemporary approach to coordinated postresuscitative care by combining MTH with coronary angiography is associated with more favorable patient outcomes $[13,22,23]$. Sunde et al. reported $56 \%$ survival with favorable neurologic outcomes for patients randomized to a standardized postresuscitation protocol involving MTH and PCI, as compared to $26 \%$ in the control treatment arm [22]. Similarly, Stub et al. demonstrated that the combination of therapeutic hypothermia with early coronary intervention was associated with an improved survival of $64 \%$, as compared to $39 \%$ in the control group [13]. The results of the current study are comparable to those previously stated. However, in our study over $80 \%$ of patients experienced cardiogenic shock within the first week after cardiac arrest and 36\% required intra-aortic balloon pump counterpulsation, which is significantly higher than 
that reported in the previous literature [13]. Unlike previous studies, we did not exclude cardiogenic shock patients from the MTH protocol $[5,6,9]$. Although circulatory shock is still considered a relative contraindication for $\mathrm{MTH}$, several recent studies including ours suggest that these patients may still derive benefit from MTH $[24,25]$.

Previous literature has identified key clinical characteristics to predict survival from OHCA: initial location of OHCA, witnessed cardiac arrest, prompt bystander CPR, ECG with shockable cardiac rhythm, early defibrillation, time to resuscitation, complete revascularization, hyperlactatemia, and presence of $\mathrm{ABI}[4,6,26-30]$. In addition, renal insufficiency is a known predictor of mortality in the cardiogenic shock population [30]. Kidney function as assessed by $\mathrm{CrCl}$ appears to be a sensitive marker of poor tissue perfusion during cardiac arrest and perhaps an indirect marker of poor cerebral perfusion. Our findings confirmed the importance of renal function as an independent predictor of both inhospital mortality and neurological outcome in the OHCA patient population who underwent $\mathrm{MTH}+\mathrm{C}$.

In addition to renal perfusion, bystander CPR improved 1-year survival with favorable neurological outcomes for OHCA patients in a study by Iwami et al. [29]. A similar study by Herlitz et al. further supported the survival benefit of bystander CPR afforded to this patient population [28]. Prompt provision of CPR may delay the degradation of tachyarrhythmias to asystole, explaining the positive impact on survival. Despite the majority of patients having experienced witnessed cardiac arrest, only half of patients had immediate bystander CPR in our population. The absence of immediate CPR was a potent independent negative predictor of good neurologic recovery, in keeping with previous studies. This stresses the critical importance of public awareness and education in early bystander CPR to improve the neurologic outcome for OHCA patients. The study population also experienced a longer median arrest time (low flow) $(28 \pm$ $15 \mathrm{~min}$ ) as compared with other studies [5, 6]. A longer interval from collapse to ROSC has been associated with unfavorable neurologic outcomes, which is corroborated by our findings [6].

Although the correct population was cooled in the current study, the time to reach target temperature $\left(32-34^{\circ} \mathrm{C}\right)$ from initiation of MTH was relatively prolonged at $8 \mathrm{hrs}$. The prolonged time to reach target temperature may be attributable to delayed initiation of the MTH protocol as a consequence of early coronary angiography, as less than $25 \%$ of our study population was cooled prior to catheterization. Of note, there was a trend towards less favorable outcomes in patients who had no cooling prior to coronary angiography, and it was a univariate predictor of in-hospital mortality and neurological outcome. This suggests the need for a well-planned and coordinated cooling protocol which is implemented prior to and during catheterization, such that early catheterization does not delay the initiation of MTH. Second, a transport based MTH protocol is required, in order to enable the implementation of MTH en route to central catheterization facilities. Finally, increased education for earlier MTH implementation in peripheral facilities (ER, ICU) will hopefully improve patient outcomes.
There are several limitations to the current study. This is a single-centre retrospective review and thus subject to potential confounders and selection bias. Data collection was limited by available documentation and not all measurements were made at exactly the same time intervals or course in hospital. We used the CPC score to assess neurologic recovery because of its ease of use and widespread reporting in the literature. However, this scoring system is not well validated and was retrospectively assigned based on clinical documentation at the time of discharge. Finally, our study does not include long-term outcome data and was limited to hospital discharge.

\section{Conclusion}

Although the outcome for patients resuscitated from cardiac arrest is traditionally low, it may be improved by the use of coordinated resuscitation protocols involving MTH and cardiac catheterization. Baseline renal insufficiency appears to be a potent predictor of in-hospital mortality and poor neurologic outcome in this population. The absence of immediate CPR also predicts poor neurologic recovery and this should be an impetus for widespread public education. Further multicentre registry collaboration may be indicated to study and refine outcomes in this severely ill patient population.

\section{Conflict of Interests}

The authors declare that there is no conflict of interests regarding the publication of this paper.

\section{References}

[1] Statistics Canada, Mortality, Summary List of Causes 2008, Statistics Canada, 2008.

[2] American Heart Association, "American Heart Association guidelines for cardiopulmonary resuscitation and emergency cardiovascular care," Circulation, vol. 112, pp. IV-1-IV-5, 2005.

[3] C. Sasson, M. A. M. Rogers, J. Dahl, and A. L. Kellermann, "Predictors of survival from out-of-hospital cardiac arrest a systematic review and meta-analysis," Circulation: Cardiovascular Quality and Outcomes, vol. 3, no. 1, pp. 63-81, 2010.

[4] J. Herlitz, A. Bång, J. Gunnarsson et al., "Factors associated with survival to hospital discharge among patients hospitalised alive after out of hospital cardiac arrest: change in outcome over 20 years in the community of Göteborg, Sweden," Heart, vol. 89, no. 1, pp. 25-30, 2003.

[5] Hypothermia after Cardiac Arrest Study Group, "Mild therapeutic hypothermia to improve the neurologic outcome after cardiac arrest," The New England Journal of Medicine, vol. 346, no. 8, pp. 549-556, 2002.

[6] N. Neilsen, J. Wetterslev, T. Cronberg et al., "Targeted temperature management at $33^{\circ} \mathrm{C}$ versus $36^{\circ} \mathrm{C}$ after cardiac arrest," The New England Journal of Medicine, vol. 369, no. 23, pp. 2197-2206, 2013.

[7] J. Hovdenes, J. H. Laake, L. Aaberge, H. Haugaa, and J. F. Bugge, "Therapeutic hypothermia after out-of-hospital cardiac arrest: experiences with patients treated with percutaneous coronary 
intervention and cardiogenic shock," Acta Anaesthesiologica Scandinavica, vol. 51, no. 2, pp. 137-142, 2007.

[8] R. Knafelj, P. Radsel, T. Ploj, and M. Noc, "Primary percutaneous coronary intervention and mild induced hypothermia in comatose survivors of ventricular fibrillation with ST-elevation acute myocardial infarction," Resuscitation, vol. 74, no. 2, pp. 227-234, 2007.

[9] S. Wolfrum, C. Pierau, P. W. Radke, H. Schunkert, and V. Kurowski, "Mild therapeutic hypothermia in patients after outof-hospital cardiac arrest due to acute ST-segment elevation myocardial infarction undergoing immediate percutaneous coronary intervention," Critical Care Medicine, vol. 36, no. 6, pp. 1780-1786, 2008.

[10] H. O. Peels, G. A. J. Jessurun, I. C. C. van der Horst, A. E. R. Arnold, L. H. Piers, and F. Zijlstra, "Outcome in transferred and nontransferred patients after primary percutaneous coronary intervention for ischaemic out-of-hospital cardiac arrest," Catheterization and Cardiovascular Interventions, vol. 71, no. 2, pp. 147-151, 2008.

[11] F. Dumas, A. Cariou, S. Manzo-Silberman et al., "Immediate percutaneous coronary intervention is associated with better survival after out-of-hospital cardiac arrest: insights from the PROCAT (Parisian Region Out of Hospital Cardiac Arrest) registry," Circulation: Cardiovascular Interventions, vol. 3, no. 3, pp. 200-207, 2010.

[12] P. Cronier, P. Vignon, K. Bouferrache et al., "Impact of routine percutaneous coronary intervention after out-of-hospital cardiac arrest due to ventricular fibrillation," Critical Care, vol. 15, article R122, 2011.

[13] D. Stub, C. Hengel, W. Chan et al., "Usefulness of cooling and coronary catheterization to improve survival in out-of-hospital cardiac arrest," American Journal of Cardiology, vol. 107, no. 4, pp. 522-527, 2011.

[14] V. B. Nanjayya and V. Nayyar, "Immediate coronary angiogram in comatose survivors of out-of-hospital cardiac arrest-an Australian study," Resuscitation, vol. 83, no. 6, pp. 699-704, 2012.

[15] P. T. O'Gara, F. G. Kushner, D. D. Ascheim et al., "2013 ACCF/AHA guideline for the management of st-elevation myocardial infarction: a report of the American College of Cardiology Foundation/American Heart Association Task Force on Practice Guidelines," Journal of the American College of Cardiology, vol. 61, no. 4, pp. e78-e140, 2013.

[16] I. Jacobs, V. Nadkarni, J. Bahr et al., "Cardiac arrest and cardiopulmonary resuscitation outcome reports: update and simplification of the Utstein templates for resuscitation registries. A statement for healthcare professionals from a task force of the international liaison committee on resuscitation (American Heart Association, European Resuscitation Council, Australian Resuscitation Council, New Zealand Resuscitation Council, Heart and Stroke Foundation of Canada, InterAmerican Heart Foundation, Resuscitation Council of Southern Africa)," Resuscitation, vol. 63, no. 3, pp. 233-249, 2004.

[17] W. L. Wright and R. G. Geocadin, "Postresuscitative intensive care: neuroprotective strategies after cardiac arrest," Seminars in Neurology, vol. 26, no. 4, pp. 396-402, 2006.

[18] V. A. Negovsky, "Postresuscitation disease," Critical Care Medicine, vol. 16, no. 10, pp. 942-946, 1988.

[19] C. M. Spaulding, L.-M. Joly, A. Rosenberg et al., "Immediate coronary angiography in survivors of out-of-hospital cardiac arrest," The New England Journal of Medicine, vol. 336, no. 23, pp. 1629-1633, 1997.
[20] L. M. Batista, F. O. Lima, J. L. Januzzi Jr., V. Donahue, C. Snydeman, and D. M. Greer, "Feasibility and safety of combined percutaneous coronary intervention and therapeutic hypothermia following cardiac arrest," Resuscitation, vol. 81, no. 4, pp. 398-403, 2010.

[21] J. C. Reynolds, C. W. Callaway, S. R. El Khoudary, C. G. Moore, R. J. Alvarez, and J. C. Rittenberger, "Coronary angiography predicts improved outcome following cardiac arrest: propensityadjusted analysis," Journal of Intensive Care Medicine, vol. 24, no. 3, pp. 179-186, 2009.

[22] K. Sunde, M. Pytte, D. Jacobsen et al., "Implementation of a standardised treatment protocol for post resuscitation care after out-of-hospital cardiac arrest," Resuscitation, vol. 73, no. 1, pp. 29-39, 2007.

[23] M. Werling, A.-B. Thorén, C. Axelsson, and J. Herlitz, "Treatment and outcome in post-resuscitation care after out-ofhospital cardiac arrest when a modern therapeutic approach was introduced," Resuscitation, vol. 73, no. 1, pp. 40-45, 2007.

[24] A. O. Spiel, A. Kliegel, A. Janata et al., "Hemostasis in cardiac arrest patients treated with mild hypothermia initiated by cold fluids," Resuscitation, vol. 80, no. 7, pp. 762-765, 2009.

[25] R. Gal, M. Slezak, I. Zimova, I. Cundrle, H. Ondraskova, and D. Seidlova, "Therapeutic hypothermia after out-of-hospital cardiac arrest with the target temperature 34-35 degrees C," Bratislavské Lekárske Listy, vol. 110, no. 4, pp. 222-225, 2009.

[26] I. Lund-Kordahl, T. M. Olasveengen, T. Lorem, M. Samdal, L. Wik, and K. Sunde, "Improving outcome after out-of-hospital cardiac arrest by strengthening weak links of the local Chain of Survival: quality of advanced life support and post-resuscitation care," Resuscitation, vol. 81, no. 4, pp. 422-426, 2010.

[27] H. Hayashi and Y. Ujike, "Out-of-hospital cardiac arrest in Okayama City (Japan): outcome report according to the 'Utstein style", Acta Medica Okayama, vol. 59, no. 2, pp. 49-54, 2005.

[28] J. Herlitz, L. Svensson, S. Holmberg, K.-A. Ängquist, and M. Young, "Efficacy of bystander CPR: intervention by lay people and by health care professionals," Resuscitation, vol. 66, no. 3, pp. 291-295, 2005.

[29] T. Iwami, T. Kawamura, A. Hiraide et al., "Effectiveness of bystander-initiated cardiac-only resuscitation for patients with out-of-hospital cardiac arrest," Circulation, vol. 116, no. 25, pp. 2900-2907, 2007.

[30] F. Hussain, R. K. Philipp, R. A. Ducas et al., "The ability to achieve complete revascularization is associated with improved in-hospital survival in cardiogenic shock due to myocardial infarction: manitoba cardiogenic SHOCK Registry investigators," Catheterization and Cardiovascular Interventions, vol. 78, no. 4, pp. 540-548, 2011. 


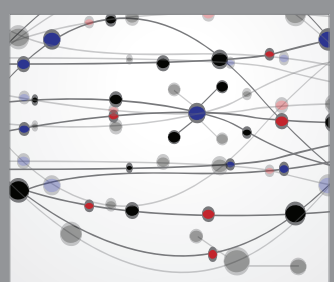

The Scientific World Journal
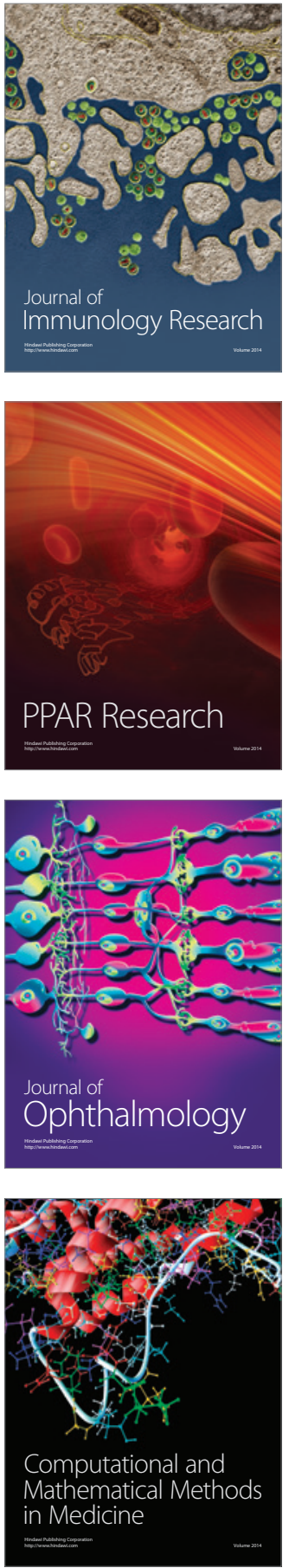

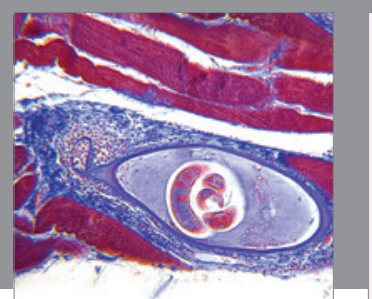

Gastroenterology Research and Practice

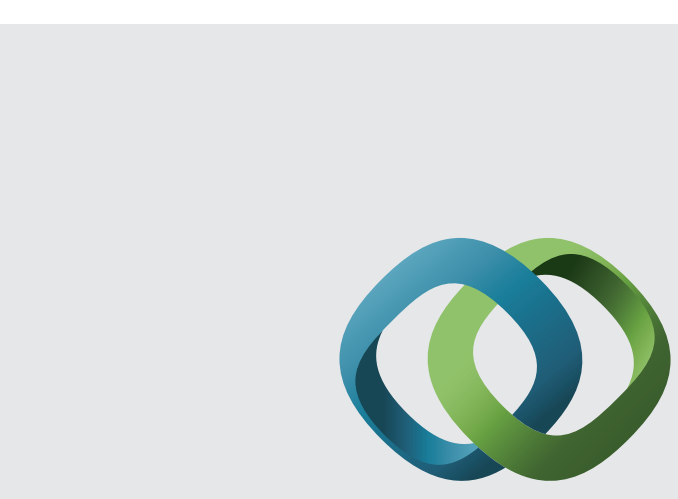

\section{Hindawi}

Submit your manuscripts at

http://www.hindawi.com
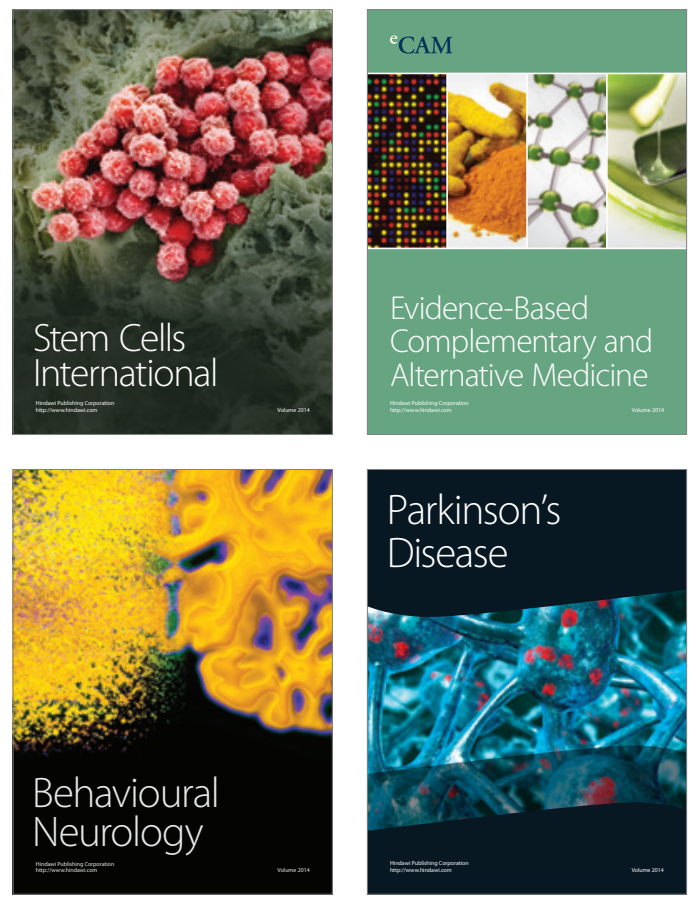
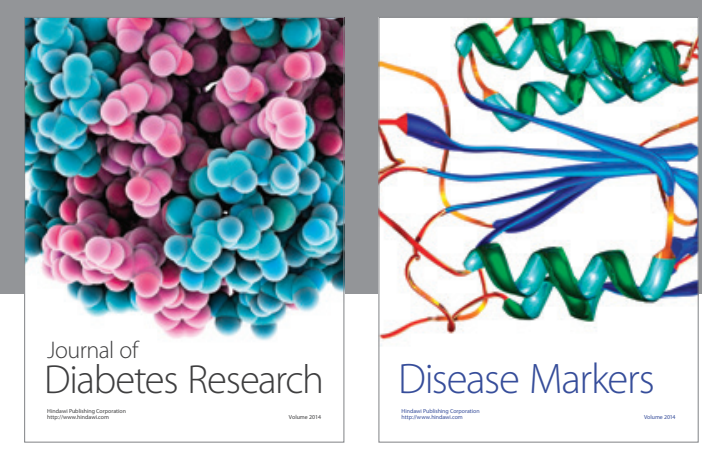

Disease Markers
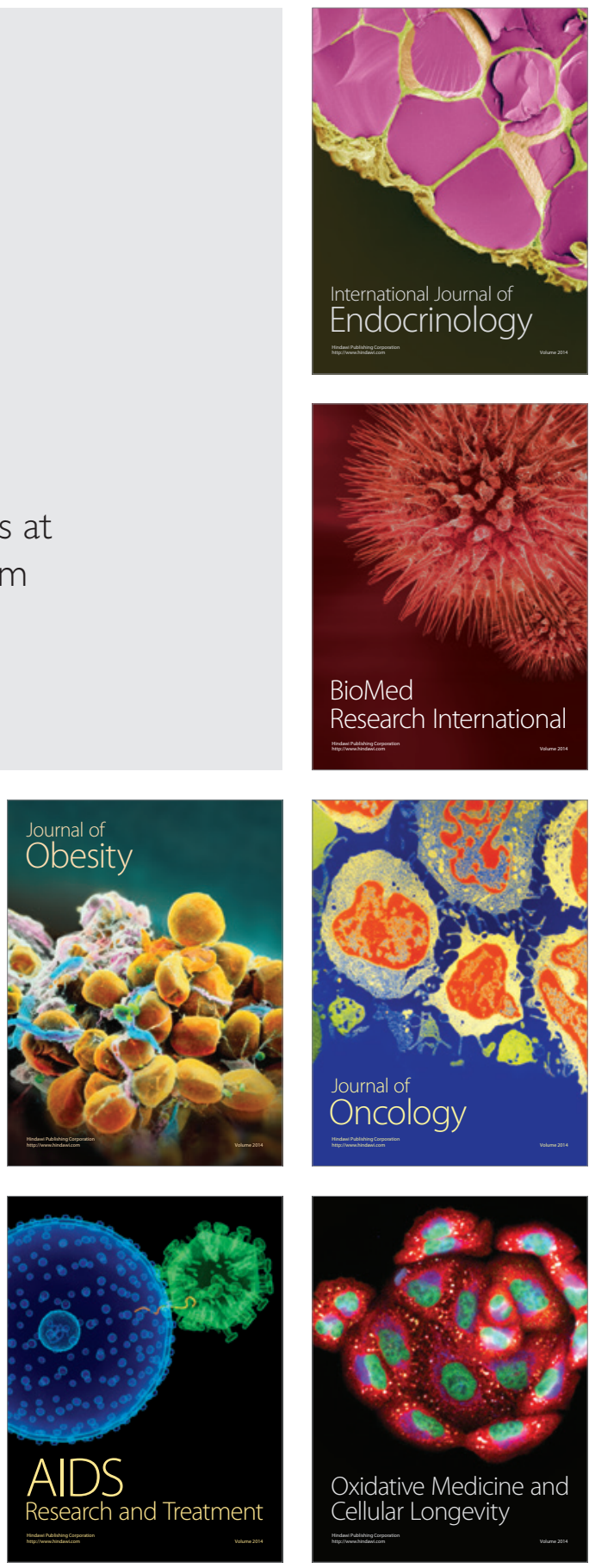